\title{
Reservorio ileal con anastomosis reservorio anal por colitis ulcerosa: Complicaciones y resultados funcionales a largo plazo
}

\author{
Alejandro Zárate $C^{1}$, Álvaro Zúñiga $D^{1}$, George Pinedo $M^{1}$, \\ Francisco López $\mathrm{K}^{1}$, María E Molina P1, Paola Viviani $\mathbf{~}^{2}$. \\ Ileal pouch-anal anastomosis \\ for ulcerative colitis: Complications \\ and long term functional results
}

Background: The ileo anal-pouch-anastomosis (IPAA) is the treatment of choice for patients with ulcerative colitis (UC). Aim: To analyze the surgical outcomes, long term evolution and functional results of IPAA. Material and methods: All patients subjected to an IPAA, from 1984 to 2006 were identified from a prospectively constructed inflammatory bowel disease database. Surgical variables, postoperative complications and functional evaluation, using Öresland score were analyzed. Chi square, Fischer exact test, T Student, Mann Whitney and binary logistic regression were included in the statistical analysis. Results: In the study period 107 patients, aged 14 to 62 years (61 females), subjected to an IPAA, were identified in this period. All patients, except 4, had a J pouch. All were protected with a loop ileostomy. Thirteen patients (12.1\%) had specific postoperative complications: pelvic collections in five (4.6\%), wound infection in four (3.7\%), fistula of the anastomosis in two (1.8\%), hemoperitoneum and pouch necrosis in one each. Three (2.7\%) patients were reoperated. There was no post-operative (30 days) mortality. A complete follow-up was obtained in 106 of 107 patients: four evolved as Crohn disease; four lost their pouch and two died for other causes. One patient required an ileostomy due to a vaginal fistula. Seventy two patients were followed more than 36 months after ileostomy closure and $92 \%$ have a satisfactory intestinal function. In the univariate analysis, poorest intestinal function was related to age of diagnosis of UC and presence of chronic pouchitis. In the multivariate analyses age of diagnosis was associated with poor function. Conclusions: IPAA has a low rate of complications. The long term intestinal function is satisfactory in most patients. A poorer intestinal function was observed in older patients and those with chronic pouchitis (Rev Méd Chile 2008; 136: 467-74).

(Key w ords: Colitis, ulcerative; Colonic pouches; Proctocolectomy, restorative)

Recibido el 28 de mayo, 2007. Aceptado el 22 de octubre, 2007.

${ }^{1}$ Departamento de Cirugía Digestiva. ${ }^{2}$ Departamento de Salud Pública. Pontificia Universidad Católica de Chile. Santiago de Chile.

Correspondencia a: Dr. Álvaro Zúñiga Díaz. Departamento de Cirugía Digestiva. Facultad de Medicina. Pontificia Universidad Católica de Chile. Marcoleta 367. Santiago. Teléfono: 3543870. Fax: 6329620. E mail: azuniga@med.puc.cl 
E s conocido que un porcentaje considerable, que bordea $70 \%$ para los pacientes con enfermedad de Crohn (EC) y $40 \%$ para los portadores de colitis ulcerosa (CU), necesitarán tratamiento quirúrgico durante su vida ${ }^{1}$. La alternativa quirúrgica de elección hoy en día para los pacientes con CU es la proctocolectomía con reservorio ileal y anastomosis reservorio anal (RIA). Si bien esta operación permite la curación de la enfermedad, expone al paciente a complicaciones propias de la cirugía y al compromiso de la función intestinal, donde la frecuencia y continencia son los parámetros más expuestos.

La operación fue descrita a fines de la década 1970-79 por A. Parks ${ }^{2}$, siendo modificada posteriormente en orden a mejorar la función evacuatoria. En este sentido, los cambios más significativos han sido los relacionados con la anastomosis ileoanal, logrando así disminuir el trauma del aparato esfinteriano y facilitando la evacuación del reservorio.

En el Departamento de Cirugía Digestiva, de la Facultad de Medicina de la Pontificia Universidad Católica de Chile, esta operación fue incorporada en 1984, por lo tanto, en nuestro caso también se han introducido estas modificaciones para mejorar los resultados funcionales ${ }^{3}$. Variadas series han descrito resultados a corto plazo ${ }^{4,5}$, sin embargo, los resultados alejados y análisis de ellos no han sido ampliamente difundidos ${ }^{6}$. Llama la atención que las series internacionales que comunican resultados a largo plazo provienen de centros con más de 1.000 pacientes operados, en cambio hay muy pocas comunicaciones de series de menos de 200 pacientes. Destaca en el medio nacional una comunicación de Jensen et $\mathrm{al}^{7}$, en la cual se presentan diferentes opciones para el tratamiento quirúrgico de la CU, incluyendo el análisis de 32 reservorios ileales.

El objetivo de este trabajo es presentar las complicaciones y resultados a largo plazo de los pacientes sometidos a RIA por CU.

\section{MATERIAL Y MÉTODOS}

En la base de datos de enfermedades inflamatorias intestinales del Departamento de Cirugía Digestiva elaborada prospectivamente, se identificaron todos los pacientes sometidos a RIA por CU en forma consecutiva desde enero de 1984 hasta diciembre de 2006.

Los pacientes fueron operados en 2 ó 3 etapas, según hubiesen tenido o no una colectomía total previa por crisis grave de CU. Así, en los pacientes sometidos a una colectomía total previa, 6 meses después se practicó la proctectomía con reservorio ileal e ileostomía en asa. Finalmente, el cierre de ileostomía se realizó 3 meses más tarde; esta modalidad corresponde a 3 etapas. A los pacientes en quienes se realizó la proctocolectomía, reservorio ileal e ileostomía en asa en un mismo acto quirúrgico y posteriormente el cierre de ileostomía, se les denomina como cirugía en dos etapas. Todos los pacientes, excepto 4, recibieron un reservorio en J. El diagnóstico de CU fue hecho por el cuadro clínico y el estudio histopatológico de las biopsias endoscópicas y confirmado por el estudio anatomopatológico de la pieza quirúrgica. Previo a la realización de un RIA, se evaluó la continencia clínicamente. De esta forma, los pacientes intervenidos tenían una continencia anal satisfactoria.

La evaluación preoperatoria incluyó exámenes generales como hemograma, perfil bioquímico, evaluación del estado nutricional mediante la albúmina plasmática, linfocitos plasmáticos y cálculo del índice de masa corporal y estudio endoscópico de recto y colon.

La preparación preoperatoria consistió en un régimen hídrico el día previo a la cirugía; preparación mecánica del colon (solución de polietilenglicol) en los que correspondiese y administración de antibióticos endovenosos una hora antes del comienzo de la operación.

Los pacientes fueron seguidos clínicamente tanto en consulta por cada cirujano tratante, como mediante cuestionarios por vía telefónica. La función intestinal fue evaluada con el test de Öresland $^{8}$, que incluye 13 parámetros con una puntuación para cada uno (Tabla 1).

La técnica quirúrgica ha sido descrita en una comunicación anterior ${ }^{2}$. Brevemente, el paciente se instala en posición de Lloyd-Davis, se realiza una laparotomía media. Se seleccionó un punto en el ileon distal, distante a $15 \mathrm{~cm}$ de la válvula ileocecal, este punto se proyectó hacia el pubis para asegurar que este extremo alcanzaría la línea pectínea. Luego se procedió a la resección del recto, manteniendo el plano de disección junto a 
Tabla 1. Evaluación funcional de reservorio ileal. Test de Ö resland. Parámetros y puntajes considerados. Función: satisfactoria 0 a 3, aceptable 4 a 6 y deficiente 7 a 16

\begin{tabular}{|c|c|c|}
\hline Parámetro & Hallazgo & Puntos \\
\hline \multirow[t]{3}{*}{ Número de evacuaciones/día } & $<4$ & 0 \\
\hline & 5 & 1 \\
\hline & 6 o más & 2 \\
\hline \multirow[t]{3}{*}{ Número de evacuaciones/noche } & 0 a una por semana & 0 \\
\hline & Más de una por semana & 1 \\
\hline & 2 o más por noche & 2 \\
\hline \multirow[t]{2}{*}{ Urgencia } & No & 0 \\
\hline & Sí & 1 \\
\hline \multirow[t]{2}{*}{ Evacuación dificultosa } & No & 0 \\
\hline & Sí & 1 \\
\hline \multirow[t]{2}{*}{ Escurrimiento/día } & No & 0 \\
\hline & Sí & 1 \\
\hline \multirow[t]{2}{*}{ Escurrimiento/noche } & No & 0 \\
\hline & Sí & 1 \\
\hline \multirow[t]{2}{*}{ Incontinencia a gases } & No & 0 \\
\hline & Sí & 1 \\
\hline \multirow[t]{3}{*}{ Irritación perineal } & No & 0 \\
\hline & Ocasionalmente & 1 \\
\hline & Permanentemente & 2 \\
\hline \multirow[t]{2}{*}{ Uso de apósitos durante el día } & No & 0 \\
\hline & Sí & 1 \\
\hline \multirow[t]{2}{*}{ Uso de apósitos durante la noche } & No & 0 \\
\hline & Sí & 1 \\
\hline \multirow[t]{2}{*}{ Restricción de la dieta } & No & 0 \\
\hline & Sí & 1 \\
\hline \multirow[t]{2}{*}{ Requerimientos de medicamentos } & No & 0 \\
\hline & Sí & 1 \\
\hline \multirow[t]{2}{*}{ Alteración vida social } & No & 0 \\
\hline & Sí & 1 \\
\hline
\end{tabular}

Nota: Noche: Después de las 23 h, hasta antes de las 6 AM.

Tabla traducida reproducida del trabajo de Öresland T, Fasth S, Nordgren S, Akervall S, Hultén L. Pouch size: the important functional determinant after restorative proctocolectomy. Br J Surg 1990; 77: 265-9. Copyright British Journal of Surgery Society Ltd. Reproduced with permission. Permission is granted by John Wiley \& Sons Ltd on behalf of the BJSS Ltd.

la pared rectal hasta alcanzar el plano de los elevadores del ano. En los 13 primeros pacientes de esta serie se seccionó el recto a $7 \mathrm{~cm}$ del margen anal y se practicó mucosectomía transanal del muñón rectal. El reservorio en $\mathrm{S}$ o en $\mathrm{J}$ se confeccionó con sutura manual y la anastomosis reservorio anal con puntos separados por vía endoanal. Posteriormente, se introdujo el uso de aparatos de sutura mecánica, para seccionar transversalmente el recto $2 \mathrm{~cm}$ sobre la línea pectínea y se procedió a construir el reservorio en forma mecánica con aparato de sutura lineal cortante. 
La anastomosis reservorio anal se practicó por vía anal con un aparato de sutura mecánica circular. Para verificar la integridad de la sutura de la anastomosis ileoanal y del reservorio, se introdujo aire o suero en el reservorio. En todos los casos, la anastomosis reservorio anal fue protegida con una ileostomía en asa en la fosa ilíaca derecha, la cual fue cerrada entre las 8 y 10 semanas después, previa comprobación mediante estudio radiológico de la indemnidad del reservorio y de la anastomosis reservorio anal.

Se consideraron complicaciones postoperatorias aquellas ocurridas hasta 30 días después de la operación y complicaciones alejadas las que se desarrollaron después de 30 días.

En los pacientes con un seguimiento mayor de 72 meses desde la cirugía del RIA, se correlacionó la función intestinal y la frecuencia de deposiciones por noche con variables pre, intra y postoperatorias.

Análisis estadístico mediante el test de Chi cuadrado, exacto de Fischer, T de Student, Mann Whitney. Para el análisis multivariado se usó la regresión logística binaria. Se consideró significativo un valor $\mathrm{p}<0,05$.

\section{Resultados}

En el período descrito, se practicaron 107 RIA en pacientes con diagnóstico de CU.

Sesenta y una de ellas de sexo femenino (57\%). La edad promedio al momento del diag-

\section{Tabla 2. C aracterísticas de la serie}

\begin{tabular}{|lr|}
\hline Variables & No \\
\hline № total de pacientes & 107 \\
Sexo femenino & $(57 \%)$ \\
Edad al diagnóstico CU (años) & 29,9 \\
Edad al RIA (años) & 34,4 \\
Reservorios en J & 104 \\
Reservorio en S & 4 \\
Abordaje: Abierto & 99 \\
Laparoscópico & 8 \\
RIA 2 tiempos & 90 \\
RIA 3 tiempos & 17 \\
\hline
\end{tabular}

nóstico de la CU fue 29,9 años (r: 4-53). En 103 pacientes se practicó un reservorio en J y en 4, en S. Mediante laparotomía en 99 casos y por laparoscopia en 8.

El tiempo promedio entre el diagnóstico de CU y el tratamiento quirúrgico fue de 45,8 meses (i: 0348). Las características de la serie se resumen en la Tabla 2 .

La edad promedio al practicar el reservorio fue de 34,4 años (i: 14-62). Cirugía en 2 tiempos se realizó en 17 pacientes (15,9\%) y en 3 tiempos en $90(84,1 \%)$.

Una o más complicaciones postoperatorias (30 días) se observaron en 34 pacientes (31,7\%). Complicaciones relacionadas directamente con el reservorio y su anastomosis al canal anal se observaron en 13 pacientes (12,1\%), que se detallan en la Tabla 3. La temporalidad de estas complicaciones fue de 5 en los primeros 20 pacientes y 8 en los siguientes 87 pacientes $(25 \%$ versus $9 \%$ respectivamente; $\mathrm{p}$ =0,051). No hubo mortalidad.

El tratamiento de las complicaciones relacionadas directamente con el reservorio o abdominales fue conservador en $77 \%$ de los casos y solamente $3(23 \%)$ pacientes fueron reintervenidos. Complicaciones alejadas (mayor a 30 días) ocurrieron en 11 pacientes (10,3\%). Las observadas con mayor frecuencia fueron reservoritis crónica en 5 (4,7\%) pacientes e íleo mecánico en 4 (3,7\%) (Tabla 4).

Se logró un seguimiento completo en 106 de 107 pacientes (99\%), de los cuales 4 (3,7\%) evolucionaron como enfermedad de Crohn: 4 $(3,7 \%)$ han perdido su reservorio (1 por fístula, 1 por enfermedad de Crohn, 1 por sepsis pélvica y otro por isquemia). Una paciente se encuentra desfuncionalizado con una ileostomía por cuadro

Tabla 3. Complicaciones postoperatorias específicas (n: 107)

\begin{tabular}{|lrr|}
\hline Tipo de complicación & № & $(\%)$ \\
\hline Colección pelviana & 5 & $(4,6)$ \\
Infección de la herida operatoria & 4 & $(3,7)$ \\
Fístula anastomosis & 2 & $(1,8)$ \\
Necrosis del reservorio & 1 & $(0,9)$ \\
Hemoperitoneo & 1 & $(0,9)$ \\
Total & 13 & $(12,1)$ \\
\hline
\end{tabular}


Tabla 4. Complicaciones alejadas ( $\mathrm{n}: 106)$

\begin{tabular}{|lrr|}
\hline Complicación & № & $(\%)$ \\
\hline Reservoritis crónica & 5 & $(4,7)$ \\
Ileo mecánico & 4 & $(3,7)$ \\
Colección abdominal & 2 & $(1,8)$ \\
Total & 11 & $(10,3)$ \\
\hline
\end{tabular}

de fístula reservorio vaginal. Dos pacientes han fallecido por causas no relacionadas a la CU.

En 72 de los 107 pacientes, se cuenta con un seguimiento mayor de 36 meses desde el cierre de la ileostomía y con su reservorio funcionante. De ellos, $66(91,6 \%)$ poseen una función satisfactoria según la escala de Öresland y 6 presentan una función regular (8,4\%). En el análisis univariado para correlacionar las variables estudiadas con el estado regular de la función intestinal, se evidencia que la mayor edad de inicio de la $\mathrm{CU}$, la mayor edad a la cirugía del RIA y la presencia de reservoritis crónica se correlacionan con peor función intestinal. En el análisis multivariado sólo existe relación estadísticamente significativa con la peor función intestinal de los pacientes con la mayor edad de inicio de la CU (Tabla 5).

Respecto a la presencia de deposiciones nocturnas, que es una de las variables que integra la escala de Öresland y constituye uno de los síntomas más molestos para los pacientes, el análisis de los 72 pacientes con más de 36 meses de seguimiento evidenció que $22(30,5 \%)$ pacientes presentan al menos una deposición por noche. Al comparar los pacientes con y sin deposiciones nocturnas mediante un análisis univariado, se demuestra que solamente la mayor edad a la cirugía del RIA se correlaciona significativamente con la presentación de deposiciones nocturnas. En el análisis multivariado de todas las variables, la mayor edad al reservorio también destaca como factor para la presencia de deposiciones nocturnas (Tabla 6).

\section{DISCUSIÓN}

El tratamiento médico de los pacientes con CU en la mayoría de los casos es efectivo, sin embargo, el tratamiento quirúrgico se hace imprescindible ante dos escenarios. El primero es la falta de respuesta al tratamiento médico de una crisis grave de la enfermedad, en cuyo caso lo más aceptado es la colectomía total de urgencia9,10. Posteriormente, en un segundo tiempo, en forma electiva se reseca el recto remanente y se construye el reservorio ileal. El segundo es la combinación del fracaso del tratamiento médico y el deterioro en la calidad de vida de los pacientes

Tabla 5. Factores relacionados con la función intestinal según escala Ö resland

\begin{tabular}{|lccc|}
\hline Factor & \multicolumn{2}{c}{ Función Intestinal } & Valor P \\
& $\begin{array}{c}\text { Regular } \\
\text { n }\end{array}$ & Satisfactoria & \\
& \multicolumn{1}{c}{ n } & \\
\hline Sexo femenino & 5 & 37 & 0,19 \\
Antecedentes mórbidos & 3 & 30 & 0,83 \\
Complicaciones intestinales preop. & 1 & 9 & 1 \\
Complicaciones perineales preop. & 1 & 3 & 0,29 \\
Complicaciones postop. RIA total & 2 & 16 & 0,63 \\
Complicaciones postop. RIA específica & 1 & 6 & 0,47 \\
RIA 3 tiempos & 5 & 58 & 0,56 \\
Edad inicio CU & 42 & 27 & 0,004 \\
Edad al RIA & 46 & 31 & 0,009 \\
Reservoritis crónica & 2 & 3 & 0,03 \\
\hline
\end{tabular}


Tabla 6. Factores relacionados a presencia de deposiciones nocturnas

\begin{tabular}{|lccc|}
\hline Factor & Deposiciones & nocturnas & Valor P \\
& Con & Sin & \\
\hline Sexo femenino & 28 & 14 & 0,54 \\
Antecedentes mórbidos & 21 & 12 & 0,32 \\
Complicaciones intestinales preop. & 7 & 3 & 0,96 \\
Complicaciones perineales preop. & 3 & 1 & 0,80 \\
Complicaciones postop. RIA total & 13 & 5 & 0,76 \\
Complicaciones postop. RIA específica & 6 & 1 & 0,42 \\
RIA 3 tiempos & 46 & 22 & 0,12 \\
Edad inicio CU & 28,9 & 34,1 & 0,08 \\
Edad RIA & 32,9 & 38,4 & 0,03 \\
Reservoritis crónica & 2 & 3 & 0,19 \\
\hline
\end{tabular}

con un curso crónico de la enfermedad. En este caso se puede practicar la proctocolectomía y el reservorio con una ileostomía de protección y en casos seleccionados sin ella ${ }^{11}$. Las series previamente publicadas demuestran las ventajas de este tratamiento quirúrgico que, además de resecar la mucosa enferma, disminuye la posibilidad de desarrollar una neoplasia colorrectal y permite una función intestinal adecuada particularmente en términos de frecuencia de evacuaciones y continencia.

El RIA, aunque es el procedimiento de elección como tratamiento quirúrgico de la $\mathrm{CU}$, se asocia de una morbilidad inherente a toda cirugía abdominal mayor. Así, en la experiencia de Fazio (Cleveland Clinic) $^{4}$ y Pemberton (Mayo Clinic) ${ }^{12}$, con 1.005 y 390 pacientes, respectivamente, la morbilidad general es de $27 \%$ y $28 \%$, respectivamente; la morbilidad específica de $11,8 \%$ y $8 \%$, respectivamente. En nuestro estudio sobre 107 pacientes, las cifras se comparan favorablemente con las citadas, destacando el bajo porcentaje de complicaciones que requieren una reintervención quirúrgica que en este grupo fue de solamente $2,8 \% 4,13,14$.

En esta serie, el reservorio siempre fue protegido mediante una ileostomía en asa, con la finalidad de disminuir el impacto séptico de una eventual filtración de las anastomosis y el desarrollo de fístulas del reservorio. Pese a esto, se observó la presencia de fístulas en dos $(1,8 \%)$ pacientes, cifra que se compara favorablemente con series con mayor número de pacientes ${ }^{4,15}$. Estas complicaciones podrían afectar negativamente los resultados funcionales del reservorio, sin embargo, tanto en series internacionales ${ }^{16,17}$ como en el presente estudio, esto no ha sido demostrado.

$\mathrm{Al}$ analizar el desarrollo de las complicaciones relacionadas al reservorio, éstas mostraron una tendencia a ser más frecuentes al inicio de la serie. Esto ocurre en operaciones complejas mientras se cumple la curva de aprendizaje y se establece y consolida la técnica quirúrgica. Además, en los primeros casos se seccionaba el recto a $6-7 \mathrm{~cm}$ sobre la línea pectínea y se practicaba la mucosectomía de ese segmento de acuerdo al técnica de Parks, lo cual dejaba una zona desnuda de mucosa que facilita una colección hemática y posterior infección.

Respecto al desarrollo de íleo mecánico a largo plazo, en nuestra serie $4(3,7 \%)$ pacientes lo han presentado y 3 de ellos fueron resueltos quirúrgicamente. En uno fue necesaria una resección segmentaria de intestino delgado. En todos los casos, la causa fueron las adherencias. Esto demuestra la necesidad de ser particularmente riguroso en la evaluación de los pacientes portadores de un reservorio ileal con cuadro de íleo mecánico, ya que la extensión de la resección intestinal puede afectar desfavorablemente la función del reservorio. Si bien el porcentaje de pacientes que 
lo ha presentado es bajo, es posible que con mayor seguimiento aumentará esta complicación, que en algunas series alcanza hasta 22\%4,12,18,19. Es posible que en el futuro el uso de la técnica laparoscópica pueda disminuir esta cifra.

Respecto a la evaluación funcional, se observó que luego de 3 años de seguimiento la mayor parte tiene una función satisfactoria según la escala de Öresland que mide diversos parámetros ${ }^{8}$. Altos porcentajes de satisfacción respecto a la función evacuatoria, también son obtenidos en centros con mayor número de cirugías ${ }^{4,6,12}$. Así, el deterioro de la función intestinal se relacionó con la mayor edad de inicio de la CU, mayor edad del tratamiento quirúrgico y la presencia de reservoritis crónica, siendo el más importante la mayor edad de inicio de la CU como factor de riesgo para una peor función intestinal a largo plazo (Tabla 4). Este análisis permite definir una función satisfactoria, para la mayoría de los pacientes, luego de un periodo de 36 meses de reservorio funcionante después del cierre de la ileostomía. En esta serie, los resultados funcionales no se ven afectados por las complicaciones postquirúrgicas, ni otros antecedentes mórbidos de los pacientes. Respecto a la presencia de deposiciones nocturnas, que es una de las variables que integra la escala de Öresland, y constituye uno de los síntomas más molestos para los pacientes, el análisis de 72 de ellos con más de 36 meses de seguimiento demostró que 22 (30,5\%) pacientes presentan al menos una deposición por noche. Al comparar los pacientes con y sin deposiciones nocturnas, mediante un análisis uni y

\section{REFERENCIAS}

1. Bemelman Wa, Dunker MS, Slors JF, Gouma DJ. Laparoscopic surgery for inflammatory bowel disease: current concepts. Scand J Gastroenterol 2002; 236: 54-9.

2. Parks AG, Nichols RJ. Proctocolectomy with preservation of the sphincter. Br Med J 1978; 2: 85-8.

3. Zúñiga A. Reservorio ileal. Rev Chil Cir 1997; 49; 708-11.

4. Fazio VW, Ziv Y, Church JM, Oakley JR, Lavery IC, MiLsom JW et aL. Ileal pouch anal anastomoses complications and function in 1005. Ann Surg 1995; 222: 120-7. multivariado, se demuestra que solamente la mayor edad a la cirugía del RIA afecta la presencia de deposiciones nocturnas.

Si bien la mayor edad al RIA implica la presencia de deposiciones nocturnas, es claro que al integrar esta variable en el contexto de una escala con otras variables estudiadas, como en el test de Öresland, se logra una función general aceptable, en la gran mayoría de los pacientes. Una cuidadosa selección de los pacientes permitirá realizar este procedimiento a personas en las cuales el resultado de esta cirugía sea favorable.

Otro hecho importante es que $4(3,7 \%)$ pacientes evolucionaron en el largo plazo como enfermedad de Crohn, cifra similar a la que se observa en otras publicaciones. De éstos, sólo un paciente ha necesitado la extirpación del reservorio; los otros tres tienen su reservorio funcionante y una de ellas con una fístula reservorio vaginal de bajo débito. Así parece que existe un porcentaje de pacientes que pese al diagnóstico clínico y anatomopatológico de CU desarrolla una enfermedad de Crohn posteriormente. Lo anterior implica un desafío aún vigente para poder identificar a estos pacientes preoperatoriamente.

En resumen, este trabajo demuestra que la mayoría de los pacientes que requieren de tratamiento quirúrgico por $\mathrm{CU}$, en los cuales se practicó un RIA, al cabo de un periodo de seguimiento de 3 años, logran una función intestinal satisfactoria, la cual se ve desfavorecida solamente por la mayor edad al diagnóstico y tratamiento quirúrgico de la CU.

5. Farouk R, Pemberton Jh, Wolff Bg, Dozois Rr, Browning S, Larson D. Functional outcomes after ileal pouchanal anastomosis for chronic ulcerative colitis. Ann Surg 2000; 231: 919-26.

6. Marcello PW, Roberts PL, Schoetz DJ Jr, Coller JA, Murray JJ, Veidenheimer MC. Long-term results of the ileoanal pouch procedure. Arch Surg 1993; 128: 500-4.

7. Jensen C, Chiong H, Garrido R, Cúneo A, Bocic G, CÁceres M et al. Resultados de la cirugía en la colitis ulcerosa ideopática. Rev Chil Cir 2003; 55: 351-5.

8. Öresland T, Fasth S, Nordgren S, Akervall S, Hultén L. Pouch size: the important functional determinant after restorative proctocolectomy. $\mathrm{Br}$ J Surg 1990; 77: 265-9. 
9. Hyman N, Cataldo P, Osler T. Urgent subtotal colectomy for severe inflammatory bowel disease. Dis Col Rectum 2005; 48: 70-3.

10. Zúñiga A, Molina M, García-Huidobro I, López F, Rahmer A, Duarte I et al. Colectomía total de urgencia por colitis ulcerosa grave. Rev Chil Cir 2004; 56: 21-6.

11. Remzi Fh, Fazio Vw, Gorgun E, Ooi Bs, Hammel J, Preen M ET al. The outcome after restorative proctocolectomy with or without defunctioning ileostomy. Dis Colon Rectum 2006; 49: 470-7.

12. Pemberton Jh, Kelly Ka, Beart Rw, Dozois Rr, Wolff BG, ILsTrup DM. Ileal pouch-anal anastomosis for chronic ulcerative colitis: long-term results. Ann Surg 1987; 206: 504-13.

13. Arai K, Koganei K, Kimura H, Akatani M, Kitoh F, Sugita A et aL. Incidence and outcome of complications following restorative proctocolectomy. Am J Surg 2005; 190: 39-42.

14. Hultén L. Problems after ileo-pouch anal anastomosis for ulcerative colitis. How can we prevent it? What can we do? Neth J Med 1994; 45: 80-5.
15. Heuschen Ua, Hinz U, Allemeyer Eh, Autschbach F, STERn J, LuCAs M ET aL. Risk factors for ileoanal J pouch-related septic complications in ulcerative colitis and familial adenomatous polyposis. Ann Surg 2002; 235: 207-16.

16. Hallberg H, Stahlberg D, Akerlund JE. Ileal pouchanal anastomosis (IPAA): functional outcome after postoperative pelvic sepsis. A prospective study of 100 patients. Int J Colorectal Dis 2005; 20: 529-33.

17. Johnson E, Carlsen E, Nazir M, NygaArd K. Morbidity and functional outcome after restorative proctocolectomy for ulcerative colitis. Eur J Surg 2001; 167: 40-5.

18. Mcintyre Pb, Pemberton Jh, Wolff Bg, Beart Rw, Dozors RR. Comparing functional results one year and ten years after ileal pouch-anal anastomosis for chronic ulcerative colitis. Dis Colon Rectum 1994; 37: 303-7.

19. Michelassi F, HuRst R. Restorative proctocolectomy with J-pouch ileoanal anastomosis. Arch Surg 2000; 135: 347-53. 